\title{
Black Light and Condensed Space: The Image of the Universe
}

\author{
Dan Howitt \\ Private Study, Harvard University \\ (Cambridge, Massachusetts, the United States of America) \\ E-mail: dth055@g.harvard.edu \\ https://orcid.org/0000-0002-1027-8917
}

The core of this essay consists of predictions about the form of the observable universe, as well as about the form of what I refer to as the "external universe" (that is, the portion of the universe that I predict exceeds the observable universe).

All of the regions of darkness of the observable universe are regions of black light illumination. Black light is visible light, in that it can be seen. Common visible light is invisible light, in that it cannot be seen. Common visible light of particular intensities can supplant the complete expression of black light of particular intensities. Black light illuminates space as black in the absence of common visible light, thereby revealing the microlimit of the observable universe; and black light illuminates the macrolimit of the observable universe as black. If the observable universe did not have a microlimit nor macrolimit, there would be blindness upon looking between constituents of the observable universe, and upon looking at black boundary-regions of the observable universe. If the observable universe did not have a microlimit, there would be blindness in the absence of common visible light. The vision of darkness is different than blindness. Space (the microlimit), or what is on the other side of space, is the source of black light. Additional sources may be black holes, and the macrolimit, or what is on the other side of the macrolimit. There may be black light of intensities that common visible light of any intensity cannot supplant the complete expression of it. Black light is what is referred to as dark energy. The only form that space can have is that of being condensed. It cannot be curved. Gravity is a phenomenon of different degrees of, and different distributions of, condensed space. Space, and the macrolimit, cannot be precisely observed, measured, nor conceived of, as they while they are in the observable universe, they are of what exceeds the observable universe, namely the external universe. The presence of a microlimit and macrolimit entails that there is an other side of those limits, namely the external universe. The condensation of space is what is referred to as dark matter. Space pervades the observable universe, including all of the constituents of the observable universe, thereby entailing that they are fundamentally composed of space. While the constituents of the observable universe are relatively separate, they are fundamentally conjoined such that the observable universe is liquid in nature. There are no precise macrolimits of any of the constituents of the observable universe. The inability to precisely observe, measure, and conceive of the microlimit and macrolimit entails that they cannot be exceeded. The microlimit and macrolimit are formally different than any of the observable universe, as is the external universe. The observable universe was caused by, and continues to be caused by, what is of the external universe. It is impossible to know when, where, and how the observable universe began. Whereas constituents of the observable universe may expand away from one another, this does not entail that space itself - that is, the observable universe itself - is expanding. Time, infinity, zero, zero-dimensional, one-dimensional, two-dimensional, more than three-dimensional, massless, nothing, and eternal are not properties of the universe.

(C) Howitt, Dan, 2019 
Keywords: Condensed space, dark matter, black light, dark energy, microlimit, macrolimit, external universe, additive linguistic alteration, subtractive linguistic alteration, antonymic linguistic alteration, conceptual dissociation, psychological dissociation

Received: September 9, 2019; accepted: October 2, 2019

Future Human Image, Volume 12, 2019: 28-52.

https://doi.org/10.29202/fhi/12/3

It is necessary to exceed the microlimit and macrolimit of the observable universe in order to observe what they are precisely, and in order to observe where the macrolimit is precisely.

The observable universe is observable due to the presence of its microlimit and macrolimit. Hypothetically: In the absence of a microlimit, and when we looked into the visual-field, there would be blindness (not darkness) between observable constituents. Moreover, and also hypothetically: In the absence of a macrolimit, there would be blindness (not darkness) when we looked between celestial constituents.

That the observable universe has a microlimit and macrolimit entails that there must be universe that exceeds it, which I refer to as "external universe".

The external universe must be formally different than the observable universe.

The formal impossibility of physically and, or, technologically exceeding the microlimit and macrolimit of the observable universe.

The external universe, or one or more features of it, or one or more entities of it, are the initial cause, and ongoing cause and sustainer, of both the form of the observable universe, and the content of the observable universe.

The illumination of space with black light.

The pervasiveness of black light.

The intermittent supplanting of black light with common, visible-spectrum-light.

The illumination of the macrolimit of the observable universe with black light.

Black light is visible light.

Black holes as a source, or the source, of black light.

Space is the microlimit of the observable universe.

Space (the microlimit) as a source of black light.

The formal immeasurability of space and the macrolimit.

The formal unobservability of what space and the macrolimit precisely are.

Without a microlimit of the observable universe, there would be blindness (rather than vision of black space) in the absence of visible-light.

Without a macrolimit of the observable universe, there would be blindness (rather than the vision of black space) when the black regions of the macrolimit are looked at.

Without a microlimit of the observable universe, the constituents of the observable universe would be fixed in location.

Quantum gravity, and non-quantum gravity, are caused by what I conceive of as "condensed space".

Space is the microlimit of all of the constituents of the observable universe. 
The form of the observable universe is liquid: All constituents of the observable universe are integrated with other constituents, and with space; while there is relative separation between the constituents, they are fundamentally seamlessly integrated.

True separation of the constituents of the observable universe would entail the presence of external universe between the constituents, which of course is not the case.

There are no precise, or actual, macrolimits of the constituents of the observable universe.

Space (the microlimit) is the fundamental constituent of the observable universe. The observable universe cannot be fundamentally composed of particles.

The concepts of zero and infinity are conceptually dissociative.

The concepts of one-dimensional and two-dimensional are conceptually dissociative, and meaningless subtractive linguistic alterations.

The concept of more than three-dimensional is a meaningless additive linguistic alteration.

The concepts of zero-dimensional, massless, colorless, etc., are meaningless antonymic linguistic alterations.

Space cannot be curved, nor warped. Only the macrolimit of space can be curved.

The concept of time as a property of the universe is conceptually dissociative. The experience of time as a property of the universe is psychologically dissociative.

It cannot be speculated with even minimal accuracy when, where, and how the observable universe (its form and content) began.

Of anything of the observable universe that can be precisely observed, measured, or inferred to exist based on precise observations and measurements of other features or phenomena of the observable universe, it is not a fundamental feature of the observable universe.

The external universe, in necessarily being formally different than the observable universe, is surely not governed by the causation that governs the observable universe: For example, as to how the microlimit of the observable universe is a three-dimensional boundary, rather than a boundary-edge of a three-dimensional realm, this formally not only cannot be physically nor technologically investigated, it cannot be conceived of.

The measurement of comparatively condensed space via measuring the extent of illumination of visible light in different regions of space.

The measurement of comparatively condensed space via measuring the speed of light through different regions of space.

Black holes likely consist of comparatively highly condensed space, and as such, likely have comparatively stronger gravity. Common visible light would, as such, travel comparatively slower in black holes, and generally near black holes. Moreover, common visible light would, if it were not for the comparatively high intensity of the black light that is likely emitted from black holes, comparatively easily illuminate regions of black holes.

In areas of less condensed space, which, as such, have comparatively less gravity, common visible light would travel comparatively faster. Moreover, common visible light would, if it were not for the comparatively low intensity of the black light that is likely emitted from space, comparatively less easily illuminate regions of less condensed space. That is, the reason that common visible light easily illuminates regions of less condensed space is that the intensity of black light in those regions of space is comparatively low.

What is referred to as "dark matter" is surely the microlimit, and different condensations of the microlimit; and what is referred to as "dark energy" is surely black light, and different intensities of black light. 
(1) It is formally impossible to determine what precisely the microlimit and macrolimit of the universe are, because in order to do so it is necessary to exceed those limits: It is only from the perspective of what is beyond those limits, or on the other side of those limits, that those limits could possibly be precisely observed and, or, precisely measured.

(2) It is impossible to determine if any observational technology or measurement technology is such that it has the capacity to determine what the microlimit and macrolimit of the universe are: It is only from a perspective that exceeds those limits that it could be assessed whether the technology succeeded at observing and, or, measuring those limits. Simply by virtue of a technology having elicited comparatively more fundamental features of the universe, and simply by virtue of the technology continuing to do so over time, does not entail that when the technology ceases to accomplish doing so - even upon the technology being improved in crucial ways - that the technology has succeeded in determining what the microlimit and macrolimit of the universe are.

(3) Notwithstanding (1) and (2), since the universe is the entirety of reality, it cannot have a microlimit and macrolimit, nor be unlimited: The presence of a microlimit and macrolimit entails that there is an other-side of those limits; and since the universe is the entirety of reality, there cannot be anything on the other sides of reality. Moreover, and as will be discussed, the concepts of unlimited, infinite, eternal, etc., are immensely dissociative, and, as such, incoherent. Moreover, there surely is an alternative to the states of being microlimited and macrolimited, and that it is something that is formally inaccessible to human experience and conceptualization.

(4) There must be a microlimit and macrolimit of the generally observable and generally measurable universe, in light of how it is generally observable and generally measurable. (Hereafter, 'generally observable and generally measurable universe' will be referred to as 'observable universe'). As will be discussed, anything that is observable and, or, measurable is so because it is microlimited and macrolimited. Moreover, and as also will be discussed, the concepts of unlimited, infinite, eternal, etc., are immensely dissociative, and as such incoherent.

(5) In light of how there must be a microlimit and macrolimit of the observable universe, there must be an external universe - that is, a universe that exceeds the microlimit and macrolimit of the observable universe: Anything that is microlimited and macrolimited is so because there is something that exceeds its microlimit and macrolimit: Hypothetically, if there was nothing that exceeds a geometric limit, there would be no basis to conceive of it as a limit.

If there is no proof, nor evidence, that there is anything that exceeds a geometric limit, and if, throughout the entirety of humanity, no proof, nor evidence, can be uncovered, this does not mean that the limit is a limit of reality.

It is of what formally cannot be observed, nor measured, and of what cannot be conceived of to be limited, and of what is not necessarily limited, that is, for us, a limit of reality.

Of our limit of reality - the external universe - it is surely neither limited, nor unlimited: There surely is an alternative to the states of being limited and unlimited, and that it is something that is formally inaccessible to human experience and conceptualization.

(6) Unlike what is surely the case of the observable universe, and as implied by (1), and as discussed from (7) onward, (a) nothing can be observed or measured of the external universe, (b) and as such, nothing that is even minimally accurate can be conceived of the external universe, and (c) nothing that is even minimally coherent can be conceived of the external universe. 
(7) All conceptions of what exceeds the microlimit and macrolimit of the observable universe are inaccurate: All that can be accurately conceived of is that there must be such realms: It would be necessary for us to exceed the limits of the observable universe in order to have a basis with which it is possible to even minimally, accurately conceptualize what such realms are.

(8) In order for us to exceed the microlimit and macrolimit of the observable universe which would entail that we physically exceed the macrolimit, and which would entail that we can exceed the microlimit by way of observation and, or, measurement - it would be necessary for us to become radically different in nature: We would not have any of the capacities with which to observe and, or, measure the observable and measurable intricacy of what was our observable universe, and instead, would have capacities with which we can only observe and, or, measure the observable and measurable intricacy of the realm that we are in, and, the fundamental intricacy of the observable universe that we left, such as its microlimit and macrolimit.

(9) It is of course the case that any region of the observable universe is replete with the microlimit of the observable universe; yet, again, it is not only impossible for us to observe or measure what it is, we cannot with even minimal accuracy conceive of even its general form, except that it is three-dimensional, spatial, and pervades the observable universe.

(10) That the observable universe is of course replete with its microlimit entails that the microlimit is of course a three-dimensional form.

(11) In light of the aforementioned, it is likely the case that we are as we are - human by virtue of the impossibility of observing and, or, measuring the microlimit and macrolimit of the observable universe, as well as by virtue of the impossibility of observing and, or, measuring the fundamental nature of other, fundamental features of the observable universe that surely exist.

(12) In light of (11), and in light of how there must be a microlimit and macrolimit of the observable universe, it is likely the case that attempts to measure the microlimit and macrolimit that approach succeeding at doing so result in the local, inward expansion of the microlimit, and the local, outer expansion of the macrolimit; and this would result in the continuation of our uncovering of what appear to us to either be comparatively more fundamental particles, fields, or other attributes, or what appear to us to be the fundamental particles, fields, or other attributes.

(13) Black is of course a color; and the black that is observed of the general region of the macrolimit of the observable universe is surely caused by the presence of a substance: If the macro-region of the observable universe did not have a limit, then the blackness that is seen of it would instead be a region of blindness: There is a significant difference between what a sighted person sees while standing in a purely dark room — namely blackness — and what a blind person observes everywhere.

(14) It is likely the case that the black that is seen of the macro-region of the observable universe is a semblance of the macrolimit of the observable universe. Moreover, and as was discussed of the microlimit, any attempts that approach succeeding at measuring it would likely result in the outer expansion of it; and this would result in our continued uncovering of what appears to us to be either comparatively fundamental boundaries, fields, or other structures or phenomena, or what appears to us to be the fundamental boundaries, fields, or other structures or phenomena. 
(15) It is possibly the case that upon becoming significantly closer to the macrolimit of the observable universe, the semblance of it that can be seen appears to be of a different color, including of course white.

(16) The totality of the microlimit of the observable universe cannot be a conglomeration of particles of a particular, general nature; and likewise, any particular region, or relative point, of the microlimit of the observable universe cannot be one particle of a particular, general nature: For anything to be observable or measurable, including of course a particle, it must be three-dimensional - that is, it must be extended in a region of relative space in all directions in order for it to be observable and measurable; moreover, such things can either be disintegrated with existing methods of disintegration, or could be so if methods are developed that could do so; and the fact that one or more, particular methods do not succeed at doing so does not entail that the particle or particles that were attempted to be disintegrated are of the microlimit of the observable universe.

(17) There cannot be zero-dimensional particles, because zero-dimensionality refers to the lack of existence. Likewise, zero is not a thing, nor a state of existence, but rather, the absence of anything; and while it is of course possible to discover the absence of something in particular, it is not possible to discover absence itself. Moreover, "zero" (zero itself), "nothingness", "weightless", "massless", "dimensionless", "frictionless", etc., are conceptually meaningless, antonymic linguistic alterations that are undergone in order to evince considerable emotion in oneself, and in order to elicit considerable emotion in others.

There cannot be massless particles, just as there cannot be empty glasses of water. "Massless particle", "dimensionless particle", etc., are the same as "no particle", or "the absence of a particle".

(18) A particle of any nature and geometry, and any other feature of the observable universe of any nature and geometry, cannot have less than, nor more than, three spatial dimensions; and statements that something has zero spatial dimensions, and that something has one spatial dimension, and that something has two spatial dimensions, are conceptually meaningless, subtractive, linguistic alterations; and a statement that something has more than three spatial dimensions is a conceptually meaningless, additive, linguistic alteration; and both alterations are undergone in order to elicit considerable emotion in oneself, and others.

Of some people, their concepts that certain things have one spatial dimension, and that certain things have two spatial dimensions, are instead disassociations from the concept of three-dimensionality: While three-dimensionality of course consists of three different dimensions, the dimensions are nevertheless dependent on one another for their existence, such that without any one of the three dimensions, none can exist; and to conceive of onedimensionality, and two-dimensionality, as existing independently is to therefore disassociate facets of reality that depend on one another from one another. Moreover, the way that such dimensionality is pictorially conceived of, and pictorially represented, is additionally disassociative. (a) A line - which of course is conceived of as one-dimensional - in addition to having length, has height, and depth: without depth, there would be nothing to rise from the spatial-field into existence; and without height, there would be nothing to extend across the spatial-field. Moreover, when a line is observed from each of its four sides, its depth can be observed; and to limit the observing of the line to only the top-view or bottom-view is negligent; and to conceive of the line as not being observable from any of its four sides is delusive. (b) Regarding a thing $X$ that has a combined length and height, and which is conceived of as having only length and height - and it of course is conceived of as two- 
dimensional - without depth, there again would be nothing to rise from the spatial-field into existence; and to limit the observation to only the top-view or bottom-view is negligent; and to conceive of $\mathrm{X}$ as not being observable from any of its four sides, is delusive; and to conceive of $\mathrm{X}$ as being perfectly flat is incoherent, due to being a conceptually meaningless, subtractive, linguistic alteration from the state of relative flatness. In both cases - (a) and (b) - the ineluctable existence of one or two other dimensions is disassociated from by way of negligent observation, and delusive pictorialization.

The concept that certain things have zero spatial dimensions, while a conceptually meaningless, but emotionally puissant, subtractive, linguistic alteration, is not a disassociation from the concept of three-dimensionality: Three-dimensionality does not include the "zerodimensional", and as such, nothing is disassociated from.

Aside: For something to exist in the observable universe, it must be spatial; and for something to be spatial, it must be dimensional; and since the "zero-dimensional" is not dimensional, there is no such thing as a "zero-dimensional" existent.

The way that "zero-dimensionality" is pictorially conceived of, and pictorially represented, while not disassociative of course, is negligent, and delusive: The point, or point-particle, of "zero-dimensionality" has depth, length, and height in the same way that was described of the "one-dimensional" and "two-dimensional"; and to limit the observation of the point or pointparticle to only the top-view and bottom-view is negligent; and to conceive of the point or point-particle as not being observable from any of its four sides is delusive.

A drawn, or mathematically expressed, point, line, and collection of lines that form a geometry of some sort, are not existents that exist independently from the spatial world. Rather, they are as is described above, and they are misconceived of as explained above, and they are misobserved as explained above. At most, they could accurately be conceived of as relatively, minimally three-dimensional. Moreover, they are minimal representatives of objects of the typical, observable universe; and such objects are of course three-dimensional.

The concept of certain existents as having more than three spatial dimensions does not of course entail dissociation of the concept of three-dimensionality, and actually is a conceptually meaningless, additive, linguistic alteration; and since the alteration does not entail dissociation of the concept of three-dimensionality, it also does not entail the production of disassociative pictorializations.

The concept of a particular kind of existent as having, in addition to its "zero-dimensionalty", or "one-dimensionality", or "two-dimensionality", or even three-dimensionality, one or more other dimensions, does not entail that the existent has one or more additional spatial dimensions, but rather, that it has one or more other properties: Properties are most accurately described as properties, rather than as dimensions, as the description of dimensions is most apposite for spatiality. It would therefore only be accurate to describe what are argued to be additional dimensions as properties. And whereas dimensions are, of course, also properties, properties are not necessarily dimensions. Aside, an alternative to the above would perhaps be to describe what are argued to be additional dimensions as "non-spatial dimensions". In this case, it is clear that what is meant by 'dimensions' is 'properties'. Also aside, perhaps the above is analogous to conceiving of a sixth sense, or an extra sensory faculty beyond the five sensory faculties: What is described to be the sixth sense, or extra sense, is not a sense, but rather, a property of the mind - that is, a property of thought, internal visualization, etc. The "sixth sense" would therefore be most accurately described as a non-sensory faculty, or a mental capacity. Therefore, conceiving of non-dimensional properties as extra dimensions, 
and non-sensory capacities as extra senses, is delusive; and publicly characterizing them as such is deceptive. Moreover, engaging in this conceptualization, and public characterization, is similar in nature to engaging in the previously discussed antonymic, subtractive, and additive linguistic alterations, and the previously discussed conceptual dissociation.

(19) Time is (a) the duration-of-existence of something in relation to the origin of that thing; and the duration-of-existence can be measured by the consistent procession of a device that provides intermittent and accretional demarcations of the duration that the device has been proceeding, or (b) the post-origin measurement of the duration-of-existence of the thing with the device.

In the absence of the aforementioned device, other phenomena can be used, such as the phenomenon of the consistent procession of the appearance of the sun, and the phenomenon of the procession of thought, especially if the procession of the appearance of the sun, or the procession of the aforementioned device, are considerably internalized by the mind.

Conceiving of time as being an independent property entails dissociating from the relationship between the duration-of-existence of something and the devices that are used to provide measurements of its duration-of-existence; and conceiving of time as being something that is independent, and something within which things exist, is, as such, delusive: Can there be such a thing as temperature measurements that are independent of any thing?: Can it be X degrees independently of any feature of the observable universe?

(20) Even if a micro-particle was uncovered that could never be disintegrated, and whose content, as such, could never be analyzed, and even if a macro-boundary was reached that could never be penetrated, or disintegrated, this does not mean that the microlimit and macrolimit of the observable universe were found.

(21) In order to observe and, or, measure what our minds precisely are, including their microlimits and macrolimits, surely this could only, possibly be done by a being that is formally, radically different than us: It is quite apparent that the mind cannot be used in order to observe and, or, coherently conceive of what it itself is, just as the people of the observable universe cannot observe and, or, measure not only the microlimit and macrolimit of the observable universe, but the microlimit and macrolimit of any of the constituents of the observable universe.

Perhaps the aforementioned problem about the mind is not only an apposite analogy for the aforementioned discussion, and forthcoming discussion, about the observable universe, but perhaps it is the most apposite analogy, and - moreover — self-evidently apposite: As is the case with the observable universe, in order for us to observe what our minds precisely are, it would be necessary for us to exit our minds; and that of course is formally impossible. And even of the hypothetical in which we can exit our minds, the hypothetical is too incoherent, because (a) the mind that is a feature of our brains - that is, the mind that we desire to precisely observe - would cease to exist, and (b) we would be no different than any mind that is external to our minds - that is, we would be no different than any other person. Moreover, even of the hypothetical in which we become a radically different kind of person, and as such have a radically different kind of mind, (a) we would no longer have the mind that we desired to precisely observe, and (b), and upon exiting our brains, the mind that is a feature of our brains would cease to exist. What would be necessary for our minds to even possibly be observed and, or, measured is the observation and, or, measurement of a radically different kind of being who is, moreover, outside of the observable universe, and not of the observable universe. 
Would we rather, in order to resolve our curiosity about our minds, cease to be of our minds, cease to be the kind of beings that we are, and cease to be in the observable universe, or would we rather endure the humiliation of being incapable of having an even minimally accurate observation of and, or, measurement of, and concept of, what our own minds precisely are?

Similar to the aforementioned, would we rather, in order to resolve our curiosity about the observable universe, cease to be in the observable universe, and cease to be the kind of beings that we are?

It is astounding that we are seemingly, formally constrained from observing, measuring, and even minimally, coherently conceiving of some of the most fundamental, and seemingly simple, facets of the universe and ourselves. But, perhaps we are what we are, and as we are, because of this.

(22) If I have a ten cubic foot tank of a particular liquid substance, and in the center of the tank released a conglomeration of relatively minute particles, which gradually dispersed, and so slowly that they would not reach the boundaries of the tank for approximately one hundred years, and if I ignored the existence of the boundaries of the tank, it is reasonable for me to infer that the tank of water itself is expanding?

(23) It is impossible to even minimally accurately infer when the observable universe emerged, and how it emerged: The only way that such questions could possibly answered is from the perspective of what exceeds the microlimit and macrolimit of the observable universe, and, in particular, the perspective of beings who are radically different from us.

(24) Aside, and a revision of (8): In addition to how it is impossible to observe and, or, measure the microlimit of the observable universe, it is impossible to observe and, or, measure the macrolimit of any object of the observable universe, notwithstanding that we have exceeded their macrolimits. (It is of course the case that objects are objects by virtue of our having exceeded their macrolimits). All that we can observe and, or, measure is a semblance of their macrolimits, just as all that we can currently observe of the macrolimit of the observable universe is a semblance of it, and just as all that we could ever observe and, or, measure of it is a semblance of it. In order to observe and, or, measure the macrolimits of objects themselves, it would be necessary to exceed the microlimit of the observable universe: It could only be from that perspective that the macrolimits of the constituents of the observable universe could be observed and, or, measured, for from the perspective of what exceeds the macrolimit of the observable universe, it is likely the case that all that could be observed and, or, measured of the observable universe is its macrolimit. Moreover, that we exceed the macrolimits of the objects of the observable universe and still cannot observe nor measure the macrolimits themselves indicates that a further exceeding (the exceeding of the macrolimit of the observable universe) would not be useful, nor necessarily entail the acquisition of an additional, observational and, or, measurement capacity with which the macrolimits of the objects of the observable universe could be observed and, or, measured.

Interestingly, by way of tactile perception, or the use of tactile instrumentation, we can indeed touch the macrolimits of the objects of the observable universe that we can touch. However, what we touch is not what we observe, nor what we measure.

(25) An additional revision of (8): (8) is actually a nonsensical hypothetical, as not only is it impossible for us to observe and, or, measure the microlimit and macrolimit of the observable universe, it is, as such, impossible for us to physically exceed the microlimit and macrolimit of the observable universe; and as such, perhaps the hypothetical could have been better expressed as that if there were beings that existed in a realm that exceeds the observable universe, and 
if they could observe and, or, measure the macrolimit of the observable universe, then they would be radically different than us.

If there were beings that existed in a realm that exceeds the observable universe, and if they were not able to observe and, or, measure the macrolimit of the observable universe, then they likely would be of the same, general nature as us.

(26) It is therefore evident that what is also necessary in order to observe and, or, measure the macrolimit of the observable universe is, in addition to a perspective that exceeds the macrolimit of the observable universe, one that is radically different than our general perspective: Being on the other side of the macrolimit of the observable universe does not necessarily entail that the limit could be observed and, or, measured.

(27) An addition to (24): Perhaps analogous to what was discussed of the macrolimit of the observable universe, perhaps it is the case that if there was no microlimit of the observable universe, we, despite having the capacity to see, would be blind: Perhaps analogous to what was discussed of the macrolimit, perhaps it is the case that the various colors of objects that we see, including of course black and white, is a semblance of our microlimit, and that without a microlimit, there would be no color, and instead, blindness.

(28) In addition to how it is surely the case that the microlimit of the observable universe could not be observed and, or, measured from the perspective of what exceeds the macrolimit, it would seemingly be impossible for there to exist an observational perspective and, or, measurement-capable perspective from what exceeds the microlimit, in light of its relative minuteness. It therefore can seemingly only be hypothesized that if it was possible for there to be an observational perspective and, or, measurement-capable perspective from what exceeds the microlimit, the microlimit could be observed and, or, measured, and the macrolimits of the constituents of the observable universe could be observed and, or, measured. Moreover, and perhaps analogous to how it would seemingly be impossible to observe and, or, measure the microlimit of the observable universe from the perspective that exceeds the macrolimit, it would surely be impossible for the macrolimit of the observable universe to be observed and, or, measured from what exceeds the microlimit of the observable universe: It is likely the case that on the other side of both limits, all that could be observed and, or, measured is the limits themselves. And for this reason, I should retract my previous discussion that the only perspective from which the macrolimits of the constituents of the observable universe could be observed and, or, measured is that of what exceeds the microlimit.

(29) The content of the observable universe of course consists of extraordinary, physical complexity; and much of the content seems to be extraordinarily, observationally elusive and, or, inexplicable. But, the observable and, or, measurable content of the observable universe seems to be secondary in importance: While it is of course important to observe and, or, measure, and describe, and explain, such content, it seems that doing so is secondary in importance to the fundamental issues of what are, and where are, the microlimit and macrolimit of the observable universe, and when and how the observable universe emerged, or began to emerge.

(30) The microlimit and macrolimit of our universe cannot be fields for the reasons that are mentioned in (16) and (18).

(31) In addition to what was previously discussed about the inward expansion of the microlimit, and the outward expansion of the macrolimit, it seems that it is likely the case that the inward expansion of the microlimit entails the creation of comparatively more fundamental particles, which may emerge as sub-structures of disintegrated particles. 
In light of how the region of the macrolimit has not yet been experimentally investigated as the apparent region of the microlimit has, and in light of how it may essentially always remain quiescent due to this, there is of course uncertainty about what would occur upon succeeding at being within close proximity of it. I would surmise that it would expand outward, and that it would continue to do so in response to all of such approaches.

(32) Of course the observable universe is replete with the microlimit; and as such, the microlimit extends to the macrolimit; and as such, the macrolimit is where the microlimit discontinues.

It is seemingly tempting to construct (a) at least a general conception that what must exceed the microlimit and macrolimit is spatial, constituted by at least some matter, etc., or (b) at least a general conception that the universe is discontinued on the other sides of the microlimit and macrolimit; but it seems that all that can be conceived of is that there must be a microlimit and macrolimit, and that there must be something that exceeds those limits.

(33) In light of the aforementioned discussions about the limits, it is surely the case that what exceeds the limits is radically, formally different than the observable universe: If there is a relative micro-realm that exceeds the microlimit of the observable universe, and a relative macro-realm that exceeds the macrolimit of the observable universe, and if such realms are of the same, formal nature as the observable universe, our microlimit and macrolimit (a) would be accessible, (b) comprised of observable and, or, measurable attributes, (c) and shared between the other realms such that what would actually be the case is that there are not three, discreet realms, but rather, one realm.

Notwithstanding the aforementioned, there is no basis to think that what exceeds the microlimit is a micro-universe of the same, formal nature as ours, and that what exceeds the macrolimit is a macro-universe of the same, formal nature as ours.

(34) It is likely the case that what exceeds the microlimit and macrolimit are combined, and, moreover, that what exceeds the microlimit is what provided, and perhaps continues to provide, the constituents of our universe, and that what exceeds the macrolimit is what provided, and perhaps continues to provide, the space in which the constituents reside.

(35) Based on the aforementioned discussions, while it is of course the case that the observable universe has either a general geometry, or an ever changing, general geometry, or a chaotic geometry, or an ever changing, chaotic geometry, it is also of course the case that it is impossible to observe and, or, measure it.

(36) "Infinity" and "zero" (zero itself, rather than the absence of X) are typically conceived of as states of existence; but in order to observe and, or, measure those states, it would be necessary to exceed the negative limit of zero, and to exceed the positive limit of infinity; and doing so would be precluded by the states themselves: Without having exceeded the negative limit of zero - that is, the side of the limit of zero that is opposite to 1, or opposite to X - and without having exceeded the positive limit of infinity - that is, the side of the limit of infinity that is opposite to infinity minus 1 , or infinity minus $\mathrm{X}$ - it would not be possible to assess whether the state of zero exists, and whether the state of infinity exists; and since, according to the general concepts of zero and infinity, there is nothing less than zero, and nothing more than infinity, there would be no possibility of exceeding the negative limit of zero, nor the positive limit of infinity.

The general concepts of infinity and zero themselves demonstrate the impossibility of the states of existence of infinity and zero; and the general concepts of infinity and zero demonstrate their own incoherence. 
(37) Such a property as space seems to exist.

If there was not space itself, then the constituents of the observable universe would be permanently fixed in their locations.

The objects of the observable universe do not displace space, or replace space, because that would entail that the objects are permanently fixed in their locations.

That objects seem to exist in space, and move through it, does not entail that they displace it, nor replace it, for the aforementioned reason.

It must therefore be the case that space is within objects, and that the presence of objects does not alter it in any way.

In order to observe and, or, measure what space is, it would be necessary to exceed space that is, it would be necessary to exceed either the microlimit of space, or the macrolimit of space - and as discussed previously, that is formally impossible.

We can observe a semblance of space by way of our unaided vision, and by way of our aided vision, namely, the regions of the relative absence of objects that are between the objects that we see. Moreover, we can experience a semblance of space by way of our existence, and by way of our movement: As spatial objects, we exist within space, and can experience ourselves as such; and when we move, we can experience ourselves moving within space. But, the aforementioned is not space itself, just as the semblance of the macrolimit of the observable universe that we can observe, and the semblance of the macrolimits of constituents of the observable universe that we can observe, are not macrolimits themselves.

(38) The goal should therefore not be to uncover the microlimit and macrolimit of the observable universe, but rather, to exceed the micro-realm of the observable universe, and to exceed the macro-realm of the observable universe. That is, the goal should therefore not be to uncover the fundamental micro-constituent or micro-constituents of the observable universe, but rather, to exceed that realm of the observable universe; and the goal should therefore not be to uncover the macro-boundary of the observable universe, but rather, to exceed that realm of the observable universe.

The aforementioned goal does not entail the goal of uncovering the microlimit and macrolimit of the observable universe, but rather, ignores that goal.

Notwithstanding that it is formally impossible to accomplish the aforementioned goal, it at least is the goal which, if accomplished, would entail at least the possibility that the microlimit and macrolimit could be observed and, or, measured.

(39) The partial disintegration of protons by way of colliding them with one another at a relatively extreme speed would seemingly not possibly accomplish the goal of exceeding the microlimit, regardless of the speed at which the protons collide with one another. It seems that the most relevant experimentation, or perhaps the only relevant experimentation, for the purpose of attempting to accomplish the goal is (a) the use of explosion technology in perhaps any region of space, or a particular kind of region of space, and the observation and measurement of not what occurs in the explosion itself, but rather, to the surrounding region of space, and (b) the use of object projection technology (or object acceleration technology) in order to project or accelerate an object or objects against any region of space, or a particular kind of region of space, and the measurement of not what occurs to the object or objects, but rather, to the space that is being subjected to the object or objects.

Regarding particle collision experimentation, what would seem to be most relevant is not what occurs to the particles that are collided, but rather, to the surrounding region of space. 
It is likely the case, though, that of the experimentation of the aforementioned methods that approaches being successful at exceeding the microlimit of the observable universe, what would result is either (a) the inward expansion of the microlimit, or (b), (a) and the creation of comparatively more fundamental particles.

(40) It is impossible to conceive of a particle as being indivisible (or disintegrable): A particle, by virtue of it being a particle — that is, a three-dimensional form — can always be conceived of as being disintegrable, regardless of whether the practical capacity exists to disintegrate the particular kind of particle that is conceived of. Moreover, conceiving of a particle as not consisting of any content (or structure) is conceptually meaningless, and actually, only a conceptually meaningless, subtractive, linguistic alteration; and it is a conceptually meaningless, subtractive, linguistic alteration of the kind that was discussed earlier; and it is moreover tantamount to conceiving of a particle-less particle.

Likewise, of any particle that is uncovered, it, by virtue of being a particle, could possibly be disintegrated, regardless of whether the capacity exists to disintegrate it.

In light of how it is of course the case that the microlimit of the observable universe cannot be a conglomeration of particles, it is possibly the case that particle disintegration that approaches the microlimit of the observable universe entails the creation of one or more subparticles of a more fundamental nature in the particle or particles that are being disintegrated; and the creation, of course, would consist of the particle or particles that are being disintegrated being conferred one or more sub-particles from what exceeds, or is on the other side of, the microlimit of the observable universe.

As has been the case for considerable time, and as will likely be the case for considerable time in the future - and possibly for as long as particle disintegration experimentation continues of the particles that are uncovered and conceived of as being the most fundamental particles of the universe, they will eventually be disintegrated into comparatively more fundamental particles; and it has been, and would continue to be, impossible to observe and, or, measure if the sub-particle or sub-particles that were uncovered were already a part of the structure of the previously uncovered particle or particles, or whether they were created (or conferred) during the particle disintegration experimentation itself.

It could of course be the case that all of the particles of the observable universe already consist of such an extraordinary accretion of sub, sub-sub, sub-sub-sub, etc., particles that particle disintegration experimentation will never, for the entirety of the particle disintegration experimentation of humanity, entail the creation of, or conferring of, sub-particles.

(41) Since the macrolimit of the observable universe is not ubiquitous, as is the case of the microlimit, and since our observation, measurement, and exploration of the macro-region of the observable universe has never yielded the presence of what seems to be a region that is even approximately close to the macrolimit, the aforementioned methods, (a) and (b), would not even possibly be effective in any way.

In case our observation, measurement, and exploration of the macro-region of the observable universe yields uncovering the presence of what seems to be a region that is approximately close to the macrolimit - for example, perhaps what appears to be an encasing boundary of the observable universe is uncovered to exist at what, at that time, is a relatively accessible distance away - and in case our observation, measurement, and exploration of the macro-region of the observable universe yields accessing what appears to be an encasing of the observable universe, it is not necessarily the case that what seems to be a region that is approximately close to the macrolimit is so, and that what appears to encase the observable universe does so. 
For example, even if what seems to be an encasing boundary is uncovered, and even if, after 1,000 years of subjecting regions of it to ever advancing, disintegrating technology, penetrating technology, imaging technology, and perhaps other kinds of technology, the regions of it still remain integrated, unpenetrated, and unimaged, this does not entail that the macrolimit of the observable universe was uncovered, because it is necessary to exceed the macrolimit of the observable universe in order to uncover where it is, and what it is. Likewise, if one or more regions of the aforementioned, encasing boundary are disintegrated, or penetrated, or imaged, such that the negative side (the other side) of the apparent, macrolimit is observed and, or, measured, and even if, on the side of the apparent limit, there are no observable and, or, measurable objects, this still does not entail that the macrolimit of the observable universe was uncovered, and actually entails that it was not uncovered.

(42) To some extent akin to what was discussed about the macrolimit of the observable universe, if hypothetically there was no microlimit of the observable universe, but still a sufficient light source, we, notwithstanding that we have the capacity to see, would be blind, because (a) there would be no substance in the observable universe, and instead, only space, and (b) the space would be a region of blindness: Without a microlimit, there would be no substance in the observable universe, and instead, only space; and with the observable universe consisting only of space, there would be no color; and without the presence of color, including white and black of course, we would be blind, notwithstanding that we have the capacity to see.

Of course the aforementioned hypothetical is immensely dissociative in nature, as it dissociates from how we, and a light source, would not exist according to an aspect of it itself; but of course it is the case that hypotheticals can be used in this way in order to consider what would be the case in circumstances that have not occurred, and in circumstances that could likely never occur, and in circumstances that could never occur.

I was considering revising the aforementioned hypothetical by stating that due to the presence of the macrolimit, we, instead of being blind, would see black; but in light of how the space between us and the macrolimit would be a region of blindness, we would not see the black semblance of the macrolimit, even if, hypothetically, the macrolimit was, for example, one millimeter in front of our eyes.

(43) "Infinite", in the sense of "infinite process", which I suppose is synonymous with "eternal process" - for example, the infinite division of particles, the infinite serial accretion of numbers, etc - is a dissociative and antonymic concept: (a) In order to know whether processes do occur infinitely, it of course is necessary to observe and, or, measure that that is the case; and that is of course impossible; (b) stating that processes could occur infinitely is false, because what is necessary in order to ascertain whether that is the case is that the processes are conducted infinitely; and that of course is impossible; and (c) stating that processes could occur infinitely if they proceeded infinitely is merely hypothetical. The concept is dissociative and antonymic in the sense that it entails that the limit — namely, what does occur — is dissociated from, and then antonymically expressed to occur without limit; and the antonymic expression is a conceptually meaningless, but an intensely, emotionally eliciting, linguistic alteration.

It is the dissociation from the limit that entails the eliciting of immense emotion; and the private use, and public use, of the antonymic expression elicits the dissociation, and concurrent emotion, in oneself and others. That is, 'infinite', 'eternal', 'limitless', etc., are emotionally puissant because they entail the aforementioned dissociation. 
Conceptual dissociation, including if what ensues is the absence of alternative conceptualization, can entail the arising of immense emotion. Likewise, psychological dissociation can entail the arising of immense emotion.

Conceptual dissociation is typically undergone in order to supersede one or more undesirable, conceptual states, and in order to acquire a comparatively more preferable, conceptual state and emotional state; and psychological dissociation is typically undergone in order to supplant the memory of, and, or, the psychological effects of, past psychologically deleterious phenomena, and, or, the awareness of present psychologically deleterious phenomena, and in order to, as such, acquire a comparatively more preferable, conceptual state and emotional state.

(44) In light of how, according to the concepts of "infinity", "infinite process", "eternity", etc., there is no limit of "infinity", "infinite process", "eternity", etc., it is of course not possible to observe and, or, measure them by exceeding them; and as such, the concepts themselves preclude the possibility of determining whether there are such properties and processes in our universe.

The aforementioned concepts are not delusive, because they firstly are incoherent.

(45) The director of the Institute for Advanced Study, Robbert Dijkgraaf, proffered the following two statements:

One of the most amazing things we discovered in science is that everything is made of small particles. ... But how small are these particles? .... And does the search ever stop?

(The Institute for Advanced Study, Article: The Smallest Particles: What Do They Reveal?, November 16, 2013)

But we kind of know that in terms of if you go shorter, shorter, shorter, that at some point at this very very end ... you can't really go smaller than that: that's kind of the smallest distance there in nature. So at some point it has to stop.

(The Institute for Advanced Study, Video: The Smallest Particles: What Do They Reveal?, 50:50-51:20, November 16, 2013)

By "smallest distance there in nature", he of course is referring to the nature of "the smallest particles", and expresses his belief that an aspect of the nature of the smallest particles that constitute every thing of the universe is that they are of such a small size that it is not possible for there to be anything smaller: "you can't really go smaller than that"; and, "that's kind of the smallest distance there in nature". He moreover believes, regarding particle experimentation, that "at some point it has to stop", and that when it does, it will have revealed the "very very end", namely, "the smallest distance there in nature". He therefore seems to believe that once particle experimentation is not capable of revealing particles that are smaller than the previously revealed ones - that is, once particle experimentation, despite being proceeded with, comes to a "stop" - the "very very end", that is, "the smallest distance there in nature", will have been reached.

The problems with the aforementioned were discussed previously.

(46) It is possible that the previously discussed macrolimit is simply an intermediary, macrolimit; and if, in the future, it is thoroughly observed and, or, measured, then it surely is. 
(47) An addition to (43): Hypothetically, if the observable universe did not have a microlimit — and as such, no objects, and no light sources — and no macrolimit, a person, notwithstanding that he or she has the capacity to see, would be blind: He or she would not see blackness, nor a black region of space, but rather, would be blind.

With our eyes open while we are in an essentially, completely dark room, if the observable universe did not have a microlimit, we would not see blackness, nor a black region of space, but rather, would be blind. And if a microlimit appeared, then we would see blackness, or a black region of space, and would notice the difference between having been blind and being able to see the black region of space.

(48) While, akin to how the observable universe must have a microlimit and macrolimit, it of course had an origin, and may, likewise, have a finality. Moreover, it may have another origin, and another finality, and so one. Moreover, of the current, observable universe, it may not be the original, observable universe. In contrast, and akin to how the external universe cannot have a microlimit, nor macrolimit, nor be unlimited, it (the external universe) also cannot have an origin, nor be "infinite". Moreover, surely there is an alternative to the state of origination, and that it is something that is formally inaccessible to human experience and conceptualization.

Since the origin of the observable universe already occurred, it of course cannot be observed, nor accurately conceived of, and only speculated about.

Since the origin of the observable universe surely consisted of creation, or origination, from that which exceeds its microlimit, and from that which exceeds its macrolimit, it is formally impossible to accurately conceive of even the general form of the creation or origination.

Of whatever is coherently conceived of - of the origination of the observable universe it is secondary to the actual origination; and the fact that something is coherently conceived of demonstrates that it is secondary.

It would be necessary to be outside of the microlimit and macrolimit of the observable universe - that is, outside of the observable universe - in order to possibly observe, and, or, measure, and as such know, or only coherently conceptualize, the origination of the observable universe; and in light of our observational, measurement, and conceptual incapacities, we surely would have to be radically different than we are in order to even possibly be able to observe, and, or, measure, and as such know, or only coherently conceptualize, the origination of the observable universe.

If we could coherently conceptualize the origin of the observable universe, and coherently conceptualize, (or observe, and, or, measure, and as such know) the microlimit and macrolimit of the observable universe, and coherently conceptualize, (or observe, and, or, measure, and as such know) the microlimit and macrolimit of the constituents of the observable universe, we would be radically different in nature than we are, and - moreover - not of the observable universe, nor a part of it, nor — as such — able to participate it.

(49) There can be only one universe of course — that is, there can be only one reality but there may be one or more observable universes, because the observable universes could be separated by external universe.

(50) Whereas the macrolimits of the constituents of the observable universe are of course different than the macrolimit of the observable universe, the microlimits of the constituents of the observable universe are the same as the microlimit of the observable universe.

(51) The microlimit of the observable universe is therefore likely space itself. And just as any, even partly observable and, or, measurable object or realm must have a microlimit and 
macrolimit, and just as there are two sides of any spatial limit, there is an other side of the microlimit of the observable universe that is opposite to the side of the microlimit where the observable universe is located; and it is only from that side of the limit that the limit could possibly be observed and, or, measured itself.

(52) In light of how it is impossible for us to observe and, or, measure, and as such, even minimally, coherently conceive of, what space is itself, no arguments can be true according to which space, in the past, had one or more properties, and, or, currently has one or more properties, and, or, must have one or more properties, and, or, may in the future have one or more properties, such as a general size, general date of origin, general date of termination, and general geometry, and curvature, expansion, disintegration, diminution, compression, etc. All that can accurately be observed, and as such, conceived of, is that space itself must pervade the observable universe, and that it, as such, is of course spatial, and three-dimensional.

(53) It is surely the case that if we could observe and, or, measure space itself, we would, ignoring for the moment that we would be a radically different kind of being, and not of the observable universe, and not a part of the observable universe, be incapable of observing and interacting with the constituents of the observable universe in a way that is even minimally similar to how we currently do. For example, our visual-fields - that is, what we observe of our visual-fields - would surely be replete with the color or colors of space; and the color or colors would surely supplant the colors of the constituents of the observable universe that we previously observed: It is surely the case that at least some of the approximate $97.5 \%$ of the electromagnetic spectrum that we cannot see encolors space; but aside from that, something can be seen only if it has color: Without color-vision (which includes the inability to see black, white, and all gradations of gray), there is blindness, notwithstanding the presence of the capacity to see. As a corollary example: If we were immensely smaller in size, and had a visual acuity that is commensurate to that size, or - if we remained of our current, general size - acquired an immensely more acute, visual acuity, we, in being able to observe space itself more precisely, would surely be incapable of observing the constituents of the observable universe that we currently observe: Our new, observable universe, while not replete with color as in the aforementioned example, would consist of a profusion of objects - colored objects of course - whose existence and phenomena would be all that we could observe; and we likely would not only not even be able to coherently speculate about the existence of larger objects and phenomena, but likely would not even have a basis to think that there is anything more than what we observe.

(54) Space cannot not be curved, except at its macrolimit. (a) The interior of any region of something, regardless of the extent to which its macrolimit is curved, cannot be curved. Rather, the interior, itself, is simply extended to the macrolimit without any shape. (b) That particular kinds of phenomena occur within the interior of something that seemingly could only occur if the interior is curved does not mean that the interior is curved, but rather, that the interior consists of constituents and phenomena, which, when they interact with each other, or with something else that enters into the interior, give the appearance that the interior itself is curved. For example: A region of water, akin to the interior of any region of something, cannot not be curved, except at its macrolimit; when various kinds of things interact with the water in various ways, and the result of the interaction is such that it seems that the water itself is curved, what of course actually occurs is that various constituents of the water, and, or, various phenomena of the water, interact with the things in such a way that it seems as if the water itself is curved. (c) As an elaboration of (b): The interior of a region of something cannot, by virtue of being 
a three-dimensional region that is extended to its macrolimit, be curved. Rather, the interior is simply extended three-dimensionally to the macrolimit without any shape. In contrast, the constituents and phenomena of the interior region of something can, in a sense, be curved: There may be regions of collections of constituents that are curved; and there may be regions of phenomena that are curved. For the aforementioned, water example, if a beam of light, or a stream of a substance, is sent into a region of water in which there is a curved current, or a whirlpool, or a collection of constituents that are curved, and if the light or substance curve with these phenomena and constituents, this of course does not mean that the water itself is curved. Moreover, the beam of light, and the stream of substance, even in the absence of the aforementioned constituents and phenomena, would, due to slowing in speed, due to traveling through a region that consists of constituents and phenomena, begin to curve, until they finally discontinue both traveling and existing. This, too, does not mean that the water itself is curved.

Pictorializations and physical demonstrations of "curved space", or "warped space", are prevalent, and consist of one or more macro-boundaries of a region of pictorialized space, or a physical form, being curved in various ways. The space, or physical form, underlying the curved macro-boundaries is not curved. Moreover, notwithstanding that such pictorializations and physical demonstrations are of the macro-boundaries of the pictorializations and forms, this is dissociated from, as it is then meaninglessly stated and believed that the interior of the entirety of the displayed space, or the interior of the entirety of the displayed physical form, are also curved: This is meaningless because when this is stated and believed, nothing is conceived of. That is, this is a purely non-conceptual, linguistic statement and belief, and is akin to the aforementioned linguistic alterations, namely the antonymic, additive, and subtractive linguistic alterations. Aside, that something can be stated, including with mathematical language, does not entail that what is stated expresses anything.

Moreover - for the above pictorialized space and physical forms - only a slice of pictorialized space is provided, and only a slice of physical form is provided; and then the top surface of the slice, along with the underlying space or physical form, is displayed. The top surfaces, which are the top macrolimits of the slices, are displayed as being curved in various ways, but usually with a half-spherical concave indention in a central region, which is stated to be caused by the presence of a spherical mass, which, for the above pictorializations, is usually shown hovering immediately over the concave indentions; and for the above physical forms, often a spherical mass is placed over the physical forms, which are such that the weight of the masses causes the spherical concave indentions in the physical forms. (a) With regard to the pictorializations, only one concave indention is displayed in most pictorializations; and when such indentations are placed around the entirety of the sphere, the result is simply a circle around the sphere, and slightly outside of the sphere. Since the pictorializations that show one indentation usually do so with a grid-pattern at the macrolimit of the region of space that is shown, and, by distending the grid-pattern at the bottom of the sphere, the circle around the sphere would consist of distended grid-squares; and the spatial region outside of the distended grid-squares would consist of grid-squares that are not distended. However, since space is a three-dimensional form, only its macrolimit could be curved, as is the case with liquids, solids, and collections of gasses. While space, as is the case with liquids, solids, and collections of gasses, may be comparatively more or less condensed in various regions, it cannot be curved, bent, warped, distended, etc., just as it is not possible to curve interior regions of liquids, solids, and collections of gasses. The likely most accurate explanation, and perhaps the only nondissociative explanation, is that space is more condensed in the regions surrounding relatively 
discrete physical existents, and less condensed elsewhere. And regarding dissociation: (b) With regard to the above discussed illustration of curved space via the use of a spherical mass that is placed over a physical form which is such that the weight of the mass causes a spherical concave indention in the physical form, this phenomenon, which is limited to the use of masses of particular weights, and physical forms of particular natures, is dissociated from, and merely assumed to occur between discrete physical existents and space. But more importantly, and similar to what was discussed above in (a), if a spherical mass is implanted in the middle of a physical form, it does not bend any region of the physical form, but rather, displaces some of it, or compresses some of it: The form, around its entire macrolimit, may distend outward slightly, due to the spherical mass occupying a region where a portion of its form was; or the form may become more condensed throughout, though especially so in the area adjacent to the spherical mass. And if the form, around its macrolimit, distends outward slightly, this does not mean that the form that is underlying the macrolimit has curved, but rather, only that the macrolimit has curved (or that is, that is has been curved).

(55) In light of how there is not a state of "nothingness", etc., and in light of how space must pervade the entirety of the observable universe, space must therefore be a physical property. And in light of how space must be a physical property, the entirety of the observable universe, from at least the spatial-perspective of the microlimit (that is, the spatial-perspective of beings who are capable of such a spatial-perspective), must be liquid in nature, or something akin in nature, and - moreover - replete with color for beings with vision.

Hypothetically, of a being who is able to see the entirety of the observable universe that comprises the being's visual-field, the being's visual-field would be replete with a conglomeration of integrated and interacting objects such that the being would not be able to see beyond what is immediately in front of the being's eyes, perhaps tantamount to what a person of the observable universe would see upon being placed into a pool of integrated and interacting opaque liquids of an immense array of colors: The person would only be able to see the various liquids that are adjacent to his or her eyes. And for the purpose of this analogy, the person, while he or she is observing what occurs, would not encounter any degradation of the typical functioning of his or her eyes and vision.

(56) That it can be stated with mathematical language that a particular point, that is located at a particular place, has zero depth, zero length, and zero height, and that particular line, that is located at a particular place, has X length, zero depth, and zero height, and that a particular, geometric shape, that is located at a particular place, has X length, Y height, and zero depth, does not necessarily mean that there are such things, or that there could be such things, and that such things can be accurately, pictorially represented, and coherently, pictorially conceived.

Devising the antonymic, mathematical-linguistic alteration of a zero-dimensional existent, and the subtractive, mathematical-linguistic alterations of one-dimensional and twodimensional existents, for the purpose of concluding that what was uncovered during various kinds of experimentation are fundamental constituents of the universe, is seemingly both selfdeceptive and publicly deceptive.

Devising ingeniously furtive, dissociative, incoherent, and negligent theory in order to conclude that one's experimentation has succeeded in accomplishing the primary goal for which it was designed is seemingly dishonest. Moreover, devising such theory for the purpose of obviating, or substantially forestalling, experimental confirmation is also seemingly dishonest. 
(57) In light of (56), and (38), it is surely the case that the observable universe is additionally replete with constituents; and of course the microlimits of the constituents is space, and that the observable universe is also replete with space.

It is surely therefore case that there are no, true macrolimits of the constituents of the observable universe, as there surely is of the observable universe itself, but rather, only relative, macrolimits that are, moreover, completely integrated with other constituents of the observable universe such that there is no, true division between any of the constituents. For there to be true divisions between the constituents of the observable universe, there would have to be external universe between them; and they would, as such, be discrete, observable universes themselves.

The previously discussed issue of whether the macrolimits of the constituents of the observable universe could be observed from the other side of the microlimit of the observable universe, or the other side of the macrolimit of the observable universe, or perhaps both, is therefore not an issue, as the constituents of the observable universe cannot have true macrolimits, but rather, only relative macrolimits.

(58) It must be the case that the microlimit of the constituents of the observable universe space - is what integrates the constituents.

(59) It is surely the case that the light-spectrum (the electromagnetic spectrum) exceeds what is currently measured to be the shortest wavelength of light, and what is currently measured to be the longest wavelength of light; and in order to know whether the current, shortest wavelength of light, and the current, longest wavelength of light, and the speculated, shortest wavelength of light, and the speculated, longest wavelength of light, are the shortest and longest of the observable universe, it would necessary to exceed the observable universe, and - moreover - become radically different in nature. It is moreover surely the case that the light-spectrum consists of additional, general kinds of light than what is currently measured to be the general kinds of light, and additional, general kinds of light beyond what are currently, generally or precisely measured to be the microlimit and macrolimit of the light-spectrum: Within the visible light-spectrum, for example, there surely is an extraordinary extent of smaller (or narrower) wavelengths of light that comprise a multitude of different, general kinds of light; and as an aside, such light would be a profusion of colors that humans have never seen, nor can — as such — imagine.

In order to measure, and as such know, the complete intricacy of the light-spectrum, it surely would be necessary to exceed the observable universe, and - moreover - become radically different in nature: Beings of the observable universe are formally constrained from precisely sensory-perceiving, measuring, and conceiving of the fundamental features of the observable universe, including their minds, and it is likely the case that from the perspective of what exceeds the observable universe, and in particular, of beings who are formally, radically different than the beings of the observable universe, the fundamental features of the observable universe could be precisely sensory-perceived (or experienced in another way), measured, and conceived of.

The microlimit wavelength of the light-spectrum, and the microlimit size (or narrowness) of each wavelength of the light-spectrum, is surely the microlimit of the observable universe.

The macrolimit wavelength of the light-spectrum is likely akin to the macrolimit of any constituent of the observable universe.

Hypothetically, of any wavelengths of the light-spectrum that reach the macrolimit of the observable universe, or which would do so, their macrolimits would be the macrolimit of the observable universe. 
What light precisely is surely could only be seen and, or, measured from the perspective of beings of the external universe. If we could see and, or, measure light itself, we surely would not be able to see and, or, measure what we do of the observable universe.

(60) Non-visible light — that is, light that does not, for humans, illuminate the relative space of the observable universe, nor the constituents of the observable universe - surely does illuminate the relative space and constituents of the observable universe, and with the expression of a profusion of colors that humans cannot see, nor imagine.

Hypothetically, for sighted beings who do not see the expression of the region of the lightspectrum that we do, and who, instead, see the expression of a different region of the lightspectrum, they would surely see the constituents of the observable universe, and, at times, the relative space of the observable universe, as being of a profusion of colors; and the colors would surely be ones that we cannot see, nor imagine.

The illumination of relative space with the expression of the colors of light is discussed in (63).

It is of course possible that some or all of the other, sighted beings of the observable universe, and some or all of the sighted beings of the external universe - if there are such beings — do see, or would see, the colors that we see of our visible-light as being different than any of the colors that we see; and the colors would of course be ones that we cannot see, nor imagine. It is also possible, of course, that our visible-light is, to some of the sighted beings of the observable universe, and to some or all of the sighted beings of the external universe - if there are such beings - non-visible light (that is, not illuminating of any features of the observable universe).

(61) Since, as discussed previously, space itself cannot have internal curvature, nor any other, internal geometric form, and can only have curvature, or other, geometric forms, at its macrolimit, gravity is likely a phenomenon of the condensation of space. And as such, of regions of space in which gravity is comparatively stronger, space is likely, comparatively more condensed. And of regions of space in which gravity is comparatively weaker, space is likely, comparatively less condensed. Perhaps analogously, a comparatively more condensed liquid would entail stronger gravity within the liquid, and a comparatively less condensed liquid would entail weaker gravity within the liquid.

The condensation of space is therefore what likely causes gravitational lensing. Moreover, the concept of condensed space provides the most coherent explanation of gravity and gravitational phenomena.

The condensation of space would be, like space itself, something that cannot be accessed in order to determine what it is.

(62) The observed blackness of any region of relative space is surely due to the presence of light, for otherwise there would be blindness instead of blackness. The black of the visualfield is surely therefore produced by the expression of one or more light-sources. (The black of the visual-field is of course not revealed by light-sources: Only the black constituents of the observable universe are revealed by light-sources).

The black of the visual-field is surely therefore light itself, and could be referred to as "black light".

Light pervades the entirety of the observable universe, in light of how it is the observable universe.

In a room whose enclosure provides the most substantial, visible-light light-blocking, and non-visible-light light-blocking, that is available, the darkness that would be seen is surely caused by light that is expressed from one or more sources that are outside of the enclosure. 
If one became blind upon entering a light-blocking enclosure, then the enclosure succeeded in either blocking the entirety of light, or a considerably greater extent of it than can be accomplished anywhere else.

Relatively pure darkness is not obtained by the relative absence of light, but rather, by the relative absence of human visible-light: The black that is seen is surely one or more other kinds of light.

Unlike our visible-light, which itself is invisible to us, but whose sources are visible to us, black light is visible to us; and in light of how black light is visible to us, the sources of black light are likely invisible to us.

The black of the black constituents of the observable universe is surely not illuminated by visible-light, due to how visible-light consists of an array of colors that does not include black. The black of the black constituents of the observable universe is therefore surely always illuminated as black; and the constituents of the observable universe that are black are seen as black only when the observable constituents that surround them, and which comprise their backgrounds, are of disparate colors, and illuminated.

It is surely the case that black holes are not objectively black; and it is likewise surely the case that there are no objective, particular colors, but rather, only that all constituents of the observable universe are colored.

It is surely the case that there are no objective, visible-light sources, but rather, only light sources that are visible-light sources to particular kinds of beings.

Given the pervasiveness, uniformity, and persistence of black light throughout what seems to be the predominance of the observable universe, it is likely the case that the source or sources of it are in the external universe: If the source or sources of it were in the observable universe, surely it would, due to it, and its sources, being subjected to phenomena of the observable universe, be at least intermittently less pervasive, uniform, and persistent in various areas of the observable universe.

Black light surely pervades the entirety of the observable universe; and if it does, portions of it would therefore be supplanted by visible-light of at least a particular, minimal intensity, as well as by one or more, particular wavelengths of visible-light of at least a particular, minimal intensity, as well as by at least a minimal illumination of the observable constituents of the observable universe whose surfaces are not black. The extent of the supplanting is, of course, partly dependent on the nature of the visual-perceiver's vision; but in general, particular intensities of the aforementioned kinds of light succeed, for the predominance of humans, at, for example, (a) minimally supplanting portions of black light to the extent that at least a semblance of most of the observable constituents of the portions of the visual-field, and the relative space between the constituents, can be seen, (b) essentially completely supplanting portions of black light to the extent that all of the observable constituents of the portions of the visual-field, and the relative space between the constituents, can be seen, and (c) not only completely supplanting portions of black light, but overriding the portions of the visual-field to the extent that the portions of the visual-field, including the surfaces of all of the observable constituents of the portions of the visual-field, appear, by way of reflection (for the constituents), and by way of illumination (for the portions of relative space between the constituents), as light-sources themselves.

Hypothetically, it would surely be the case that upon the removal of all of the content of the observable universe - except the microlimit and macrolimit — the supplanting of black light would be discontinued, and black light would, as such, be completely pervasive; and this indicates that the source or sources of black light are in the external universe. 
(63) The feature of the observable universe that is either the black macrolimit of the observable universe, or which in part consists of it, may be the source of the black light of the observable universe.

(64) Instead of, "The black of the visual-field is surely therefore light itself, and could be referred to as "black light"", the black of the visual-field may be the illumination of the microlimit of the observable universe - that is, the illumination of space itself; and the source of the illumination may be the macrolimit of the observable universe, or an aspect of it.

Since the microlimit of the observable universe must be physical in nature, it is surely the case that the black-illumination of the relative space of the observable universe is the illumination of the constituent, or constituents, that comprise the microlimit.

It is of course possible that black light, the black microlimit, and the black macrolimit, are not objectively black, but rather, black only to us, and perhaps most, or all, visually-perceiving beings of the observable universe.

It is also possible that the source or sources of black light are located in the other side of the microlimit of the observable universe.

It is also possible that the source of black light is the microlimit itself.

It cannot be the case that space itself is black apart from any illumination of it: Hypothetically, if the content of the observable universe was removed - except of course the microlimit and macrolimit - and if, as such of course, there was a discontinuation of any sources of visiblelight, and if there were no sources of light in the external universe, and if the microlimit and macrolimit were not sources of light, and if the microlimit and macrolimit did not in part consist of one or more sources of light, there would, for visual-perceiving beings, be blindness, rather than darkness.

(65) An addition to (22): In order to observe and, or, measure whether the form of observable universe (the microlimit and the macrolimit) is expanding, or diminishing, and likewise, in order to observe and, or, measure whether the observable universe itself is moving, it would be necessary to exceed the observable universe.

While it can be coherently speculated that the content of the observable universe, or, a portion of the content of the observable universe, may have been of a general size, at a general time, in the past, and may be of a general size currently, and may be of a general size, at a general time, in the future, this is irrelevant to what the general size of the form of the observable universe may have been at that time in the past, and may be currently, and may be at a general time in the future.

It cannot be coherently speculated that the form of the observable universe was of a general size, at a general time, in the past, and is of a general size currently, and will be of a general size, at a general time, in the future.

In light of what was discussed previously about "infinity", "eternal", etc., the observable universe - both its content, and form - surely did have an origin; and there may have been different origins for each — that is, the form of the observable universe may have originated first, rather than both concurrently.

(66) Of anything of the observable universe that can be precisely observed and, or, measured, or inferred to exist based on precise observations and, or, measurements of other features or phenomena of the observable universe, it is not a fundamental feature of the observable universe: Anything that can be precisely observed and, or, measured could possibly be physically investigated via penetration, disintegration, etc., thereby revealing that it is not of a fundamental nature; and anything that is inferred to exist based on precise observations and, 
or, measurements of other features or phenomena of the observable universe would be of the same formal nature, though perhaps comparatively more simple, as the features or phenomena that the inference is based on.

(67) The external universe, in necessarily being formally different than the observable universe, is surely not governed by the causation that governs the observable universe: For example, as to how the microlimit of the observable universe is a three-dimensional boundary, rather than a boundary-edge of a three-dimensional realm, this formally not only cannot be physically nor technologically investigated, it cannot be conceived of.

(68) What are referred to as "black holes" are likely a cause, or the cause, of black light.

(69) Black light is visible light.

Black light can be supplanted by a sufficient extent of light that is of what is commonly referred to as the "visible light spectrum".

(70) The microlimit and macrolimit of the observable universe, while of course physical in nature, are not of the observable universe, in the sense of (a) not being the same in general nature as the features of the observable universe, namely, conceivable, analyzable, potentially analyzable, precisely observable, potentially precisely observable, etc., and (b) not being caused by, sustained by, nor affected by, the features of the observable universe.

Again, there are no macrolimits of the constituents of the observable universe.

The microlimit and macrolimit of the observable universe, while of course physical in nature, must be formally different than any feature of the observable universe.

(71) An experiment that may in part demonstrate that one region of space is more condensed than another region of space may be that of subjecting the different regions of space to the same extent of the aforementioned, visible light spectrum illumination, and assessing whether there is a difference in the extent that the regions of space and, or, the constituents of the regions of space, are illuminated.

I speculate that black holes predominately or entirely consist of comparatively highly condensed space, and that they, as such, have comparatively extremely strong gravity.

I speculate that due to the highly condensed space of black holes, it hypothetically would be relatively easy to illuminate regions of black holes with the aforementioned visible light. However, black holes are completely and intensely illuminated with black light; and the black light surely supplants the aforementioned visible light, analogous (but opposite) to how, on earth, minimal amounts of visible light supplant black light. And again, black holes are likely the sources of their own illumination.

Visible light would travel faster in regions of less condensed space, and as such, it would illuminate those regions comparatively less. However, since the black light of comparatively less condensed space is less intense, the black light is easily supplanted by visible light.

(72) As for what of black holes would produce black light: Since it is likely the case that there are no features of black holes except for (a) that they are composed of space, and, as such, replete with the microlimit of the observable universe, and (b) that they consist of comparatively highly condensed space, it is likely the case that the source of the black light is from (1) what exceeds the microlimit of the black holes, or (2) the distinctive space (that is, the distinctive microlimit) of the black holes. Regarding (2): Whereas, as was discussed earlier, all of space (the microlimit) may express black light, and in so doing, illuminate itself, regions of space that are comparatively highly condensed may express black light of greater intensity than regions of space that are comparatively less condensed. 
Black holes could be conceived of as space stars, or black stars. The region of space is the star.

Relatedly: The entirety of the observable universe must be one physical unit, as, again, there are no macrolimits of any of the relative constituents and phenomena of the observable universe. The entirety of space, therefore, is likely of one black star; and different regions of space, due to being condensed differently, would express different extents of black light.

The entirety of the observable universe is, therefore, likely one black star (or one black microlimit star). And the relative constituents and phenomena of the observable universe are likely constituents of the star.

(73) Another experiment that may in part demonstrate that one region of space is more condensed than another region of space may be that of subjecting the different regions of space to the same extent of the aforementioned, visible light spectrum illumination, and assessing whether there is a difference in the speed that the light travels through the regions of space. Where space is comparatively less condensed, and where, as such, gravity is comparatively weaker, light would travel faster than it would in regions of space that are comparatively more condensed, and where, as such, gravity is comparatively stronger.

(74) What is referred to as "dark matter" is surely the microlimit, and different condensations of the microlimit; and what is referred to as "dark energy" is surely black light, and different intensities of black light.

(75) While common visible light can supplant black light, black light is still present, as is demonstrated by how a container that blocks $100 \%$ of common visible light, and which is surrounded by profoundly intense common visible light, consists of $100 \%$ black light inside. If common visible light completely supplanted the transmission of black light across the visual field, then for someone inside the aforementioned container, there would be complete blindness (which, as discussed before, does not entail black-vision).

It may be the case, though, as was discussed, that space is a, or the, source of black light.

(76) Black light is visible light (that is, it itself can be seen), whereas common visible light is invisible (that it, it itself cannot be seen).

Black light is surely a facet of the light spectrum.

Since black light is observable, if its sources are black holes, it surely could be measured, since the sources would be of the observable universe. If black light is only a feature of the microlimit of the observable universe, and, or, the macrolimit of the observable universe, and, or, the external universe, then it surely could not be measured, because these aspects of the universe are not of the observable universe, as was discussed.

There are things in the observable universe that can be imprecisely observed, but which cannot be measured, because they are not of the observable universe: For example, space and the macrolimit of the observable universe, via black light illumination; and perhaps black light itself. The condensation of the space, itself, cannot be observed; and any measurements of it would consist of ascribing measurements to regions of space based on what occurs to various existents in those regions of space relative to what occurs to those existents in other regions of space. For example, if light slows to $\mathrm{X}$ degree, and, or, curves to $\mathrm{X}$ degree, in a particular region of space, then the region of space could be ascribed a measurement that represents this. However, the condensation of space, itself, cannot be measured. 